\title{
Non-surgical periodontal therapy improves oral health-related quality of life
}

\author{
Ruby M. S. Wong ${ }^{1}$, Sam K. S. Ng$^{2}$, Esmonde F. Corbet ${ }^{1}$, W. Keung Leung ${ }^{1 *}$ \\ ${ }^{1}$ Faculty of Dentistry, The University of Hong Kong, ${ }^{2}$ Clinical Psychology, Prince of \\ Wales Hospital, Hong Kong SAR, China.
}

Running title: non-surgical therapy improves OHQoL

Key words: Periodontitis; psychosocial aspects; quality of life; root planing;

\section{*Corresponding author:}

W. K. Leung, Room 1B25, 34 Hospital Road, Prince Philip Dental Hospital, Faculty of Dentistry, The University of Hong Kong, Hong Kong SAR, China. Telephone: +852-28590417; Fax: +852-28582532; E-mail: ewkleung@hkucc.hku.hk

Date of resubmission: August 30, 2011

\section{Conflict of interest and sources of funding statement}

The authors declare that they have no conflict of interests.

The work described in this paper was partially supported by grants from the Research

Grants Council of the Hong Kong Special Administrative Region, China (HKU 772110M) and The University of Hong Kong Research Committee grant: 10208714. 
Wong RMS, Ng SKS, Corbet EF, Leung WK. Non-surgical periodontal therapy improves oral health-related quality of life. J Clin Periodontol

\section{Abstract}

Aim: The influence of non-surgical periodontal therapy on oral health-related quality of life (OHQoL) was investigated.

Methods: 65 Chinese adults (25 males, mean 47.4 years) with moderate-to-advanced chronic periodontitis were recruited. All received oral hygiene instructions (OHI), and non-surgical periodontal treatment in a quadrant-wise approach, followed by recalls at 1, 3, 6, 9 and 12 months post-treatment, when $\mathrm{OHI}$ and prophylaxis were repeated. Clinical parameters were recorded and oral health impact profile short form (OHIP-14S) was administered at all time points.

Results: Moderate-to-deep sites $(\geq 4 \mathrm{~mm})$ decreased from $31.0 \%$ to $3.0 \%$ at 12 months post treatment $(p<0.005)$ which corresponded well with reductions in plaque, $72.8 \%$ to $25.4 \%(p<0.005)$, and bleeding on probing, $86.3 \%$ to $32.0 \%(p<0.005)$. Median OHIP-14S scores gradually reduced from 17 at baseline to 14 over the first 6 months and remained plateaued at 12 -month post-treatment $(p<0.005)$. Improvements in subdomains of physical pain, psychological discomfort and psychological disability 
accounted for the changes.

Conclusion: This study demonstrates that OHQoL, in particular pain and psychological subdomains, improvement was associated with non-surgical periodontal therapy responses. Clinicians could capitalize upon the positive psychological OHQoL impacts of mechanical periodontal treatment for subsequent patient-centered motivation during maintenance therapy.

\section{Clinical relevance}

Scientific rationale for the study: Effectiveness of non-surgical periodontal treatment is established whereas longer term impact of it upon oral health-related quality of life (OHQoL) remained to be reported. Principal findings: OHQoL improves alongside periodontal healing, in particular the OHIP-14S subscales of physical pain, psychological discomfort and psychological disability. Practical implications: Modest improvement in OHQoL is associated with periodontal healing following non-surgical periodontal therapy in patients with moderate-to-advanced periodontitis. However, the therapy failed to reverse perceived physical disability brought about by the disease destruction. Improved psychological OHQoL subdomains after treatment, however, support the relevance of patient-centered motivation in periodontal treatment. 
The era of patient-centered approaches in the health context began as early as 1948, when the World Health Organization advocated emphasis on the well-being of the patient, and not just absence of disease, in their definition of health (WHO 1948). The term "quality of life" has had varied definitions and has been described as an abstract phenomenon (McGrath \& Newsome 2007). The lack of consensus is due to different focuses under different conditions when the term "quality of life" is employed (Farquhar 1995). Thus, "oral health-related quality of life" (OHQoL) focuses on the impact of patient-perceived oral health conditions on the overall quality of life (Slade \& Spencer 1994, McGrath \& Bedi 2001, Allen 2003). Over the years, a number of instruments have been developed to assess OHQoL, including, for example, the General (previously Geriatric) Oral Health Assessment Index (GOHAI) (Atchison \& Dolan 1990), the Oral Health Impact Profile (OHIP) (Slade \& Spencer 1994), the United Kingdom Oral Health-related Quality of Life measure (OHQoL-UK) (McGrath \& Bedi 2001), and the Oral Impacts on Daily Performance (OIDP) (Adulyanon \& Sheiham 1997). The instruments typically evaluate four major aspects of OHQoL including functional limitations, pain and discomfort, psychological impacts and social or behavioral impacts (Locker et al. 2001) while OIDP and OHQoL-UK also record some other independent impacts. OHQoL-UK is able to register OHQoL in relation to both disease and health status 
(structure-function-activity-participation model) while the remaining instruments

register negative aspects related to disease status only (disease-impairment-disability-handicap model) (McGrath \& Bedi 2002).

The relationship between oral health and the quality of life has been speculated upon (Giddon 1987) based on Maslow's hierarchy of needs (Maslow 1970). Giddon considered the mouth to be essential in satisfying human biological and social needs in terms of survival, socialization and self-fulfillment, and recognized the mouth and its health as an integral part of the entire body and overall health (Giddon 1987).

Periodontal disease, with a prevalence of $15-17 \%$ among the Hong Kong adult population (Dental Service Head Office 2002) and 5-36\% of the adult population of the United States (Oliver et al. 1998), is a major oral health problem. Symptoms of periodontal disease, including redness of the gums, bleeding on brushing, loosening of teeth and halitosis, all of which can be significant oral health problems from an individual's point of view ( $\mathrm{Ng} \&$ Leung 2006a). Cross-sectional studies have shown that the impact of oral health on the quality of life in periodontal patients is high (Needleman et al. 2004) and that periodontal attachment loss contributed significantly towards poor OHQoL even after adjustment for age, gender, tooth loss and effects of caries (Ng \& Leung 2008) and/or socioeconomic position (Bernabé \& Marcenes 2010). However, clinical studies have mainly focused on therapeutic outcomes in 
terms of clinical parameters such as gain in probing attachment level (PAL) and decrease in probing pocket depth (PPD). Little attention has been paid to studying the impact of periodontal therapy from patients' perspectives. To demonstrate that periodontal therapy improves life quality requires a longitudinal study following a patient group before and after therapy (Needleman et al. 2004). One week to six months short term intervention studies (D'Avila et al. 2005, Bajwa et al. 2007, Ozcelik et al. 2007, Åslundet al. 2008, Jowett et al. 2009, Saito et al. 2010, Tsakos et al. 2010) have reported improvements of OHQoL shortly after delivery of non-surgical periodontal treatment. In addition to the short follow-up study durations, the above investigations, however, did not follow in detail how the OHQoL and its individual subscales improved with time. The aim of the present case series was therefore to follow up the healing response of chronic periodontitis patients after non-surgical periodontal treatment, and to evaluate the changes in oral health-related quality of life among this group of subjects before and for up to 12 months after a course of non-surgical periodontal therapy. The null hypothesis of the study was that there would be no change in the OHQoL of the periodontitis subjects before and after non-surgical periodontal therapy. 


\section{Materials and methods}

\section{Subjects}

This prospective case series targeted at recruiting about 60 non-smoking Chinese adults from the Reception/Primary Care Unit of Prince Philip Dental Hospital, The University of Hong Kong. The sampling approach employed a convenient sample strategy trying to recruit as many subjects as practically feasible as well as the resource and logistics consideration in the local setting for a longitudinal case series study. No formal power analysis was performed. The sample size was designed primarily based on the data from an earlier 6-month study (Bajwa et al. 2007) which had indicated that a similar number of consecutive referred periodontitis subjects could potentially satisfy the required sample size for change in OHQoL assessments after periodontal therapy. 65 subjects were recruited over a nine-month period according to the following inclusion criteria: 1) age between 35 and 65 years; 2) non-smoker; 3) a minimum of 15 teeth excluding third molars and teeth planned for extraction; and 4) at least 2 sites of $\geq 5 \mathrm{~mm}$ PPD in each quadrant. The exclusion criteria were 1) presence of conditions suggesting a need for antibiotic prophylaxis prior to periodontal examination and invasive dental treatment; 2) history of systemic disease or taking medications known to be associated with periodontal conditions; and 3) history of periodontal treatment except oral hygiene instructions, or antibiotic 
therapy in the past 6 months.

Subjects were first invited to a screening examination in which information of the study was delivered both verbally and through an information sheet. Once identified as a suitable subject, written consent was obtained, followed by study impressions for the fabrication of upper and lower periodontal examination stents.

\section{Clinical parameters}

All clinical parameters were recorded throughout by one examiner (R.M.S.W.). In the screening visit, subjects were examined for their dental and periodontal status; radiographs, study impressions were taken as necessary. PAL of all subjects at screening visit was measured from the cement-enamel junction. A detailed baseline periodontal examination was carried out approximately one week after recruitment. Clinical periodontal parameters recorded included plaque $(\mathrm{Pl} \%)$, bleeding on probing (BOP\%), PPD and PAL. Measurements were taken at 6 sites per tooth (except third molars), with the sites being mesio-buccal, mid-buccal, disto-buccal, mesio-lingual, mid-lingual, and disto-lingual aspects of each standing tooth. The PPD and PAL were measured with an electronic probe system (Florida Probe ${ }^{\circledR}$ Co. Gainesville, FL, USA), using a custom-made poly-ethylene occlusal stent with $1 \mathrm{~mm}$ thickness as a reference guide for PAL change determination from the stent edge, with guide marks to 
facilitate reproducible probe placement for PPD and PAL recordings.

\section{Study design}

Figure 1 summarizes the flow of the present study. Non-surgical periodontal therapy, including oral hygiene instructions, supra-gingival scaling, subgingival scaling and root debridement under local anaesthesia, was performed over 4-6 visits and completed within a 4-week period. These non-surgical periodontal treatments were carried out by a group of experienced dental hygienists while at the end of the last dental hygienist treatment appointment the same attending clinician (R.S.M.W.) independently clinically assessed the quality of the hygienists' care to ensure the completeness of the non-surgical periodontal therapy as described previously (Wan et al. 2009).

The psychosocial instrument used was the Oral Health Impact Profile (Chinese short-form OHIP-14S) (Wong et al. 2002). OHIP-14S includes a total of 14 questions, two for each of the seven domains (functional limitation, physical pain, psychological discomfort, physical disability, psychological disability, social disability, and handicap). Questions include asking subjects how often have they experienced difficulty in chewing, trouble pronouncing words, uncomfortable to eat, sore spots, worried or feeling miserable due to dental problems, less flavor in food, interrupted 
meals, feeling upset or being embarrassed, avoiding going out or experiencing trouble getting on with others, and unable to function or work because of dental problems. It was administered at the following time points: before commencement of non-surgical periodontal therapy, immediately after completion of the non-surgical periodontal therapy, then at 1, 3, 6, 9 and 12 months post-treatment. While the standard time reference of OHIP-14S often applied has been 6 months, the test was repeated at each follow-up visit to allow a close and continuous monitoring of the QoL changes throughout the healing and resolution period following a course of non-surgical periodontal therapy.

Patients were recalled at 1 month and 3 months after the last session of the course of non-surgical periodontal therapy, and then every other 3 months thereafter. At each of these recall visits, they received oral hygiene reinforcement and rubber-cup prophylaxis. At the subsequent 3-monthly recall visits, assessments of $\mathrm{Pl} \%, \mathrm{BOP} \%$, PPD and PAL were repeated. Thereafter, oral hygiene reinforcements were delivered and debridement was also carried out at each of these 3-month recall visits by the examiner (R.M.S.W.) as necessary. At the end of the study period, appropriate further periodontal treatment, including periodontal surgery would be prescribed for those subjects who still had sites with residual PPD $\geq 6 \mathrm{~mm}$ with BOP. 


\section{Data analysis}

Statistical analyses were performed using the statistical software package SPSS 16.0 (SPSS, Chicago, IL). As the normality (Kolmogorow-Smirnoff test) and homoscedasticity assumptions (Levene test) of the following data appeared to be valid, clinical parameters, including changes in PAL, PPD, P1\% and BOP\% were analyzed using repeated measures ANOVA. The change of OHIP-14S score and the respective seven domains: functional limitation, physical pain, psychological discomfort, physical disability, psychological disability, social disability and handicap, on the other hand, were analyzed using Friedman test, with the significance level set as $\alpha<$ 0.05 .

Clinical parameters were summarized as 1) $\mathrm{Pl} \%$, 2) $\mathrm{BOP} \%$; 3) full-mouth mean PPD, 4) full-mouth mean screening PAL, and 5) change in PAL. Clinical sites were further categorized according to the baseline measurement as shallow pockets (PPD $\leq 3 \mathrm{~mm})$, moderate pockets $(\mathrm{PPD}=4-5 \mathrm{~mm})$, and deep pockets $(\mathrm{PPD} \geq 6 \mathrm{~mm})$.

\section{Ethics}

The study protocol was approved by the Ethics Committee, Faculty of Dentistry, The University of Hong Kong (1/8/12d). 


\section{Results}

All 65 subjects (25 males) completed the whole course of non-surgical periodontal therapy and attended for recalls during the observation period over the following 12 months, as well as completing the psychosocial measure OHIP-14S administered at the assigned time points. The mean age of the 65 subjects was $47.4 \pm 6.8$, range $35-65$ years. They had a mean of $24.2 \pm 2.9$ teeth and a total of 9438 examined sites. Mean PAL at screening was $4.0 \pm 1.0 \mathrm{~mm}$, indicating severe level of attachment loss $(\mathrm{Ng} \&$ Leung 2006b).

\section{Therapeutic outcomes}

Figure 2 and Table 1 summarize the overall therapeutic outcomes in the subjects from baseline to 12 months post-treatment. Mean $\mathrm{Pl} \%$ and $\mathrm{BOP} \%$ significantly decreased from $72.8 \%$ and $86.3 \%$ at baseline to $25.4 \%$ and $32.0 \%$ at 12 months post-treatment respectively. The overall mean PPD dropped from $3.3 \pm 0.7 \mathrm{~mm}$ at baseline to $1.8 \pm$ $0.2 \mathrm{~mm}$ at 12 months with a mean gain of $0.5 \pm 0.6 \mathrm{~mm}$ in PAL. The proportion of deep pockets ( $\geq 6 \mathrm{~mm}$ ) declined from $11.2 \%$ at baseline to $0.8 \%$ at 12 months.

For initially shallow pocket sites (PPD $\leq 3 \mathrm{~mm}, \mathrm{n}=5938$ ), mean PPD decreased from $2.2 \pm 0.3 \mathrm{~mm}$ at baseline to $1.5 \pm 0.2 \mathrm{~mm}$ at 12 months, with an overall mean 
gain in PAL of $0.2 \pm 0.6 \mathrm{~mm}$. Sites with initially moderate pockets (PPD 4-5 mm, $\mathrm{n}=$ 2441) showed a drop of mean PPD from $4.4 \pm 0.2 \mathrm{~mm}$ at baseline to $2.1 \pm 0.4 \mathrm{~mm}$ at 12 months, the mean PAL gain in these sites being $0.9 \pm 0.7 \mathrm{~mm}$. As for initially deep pocket sites (PPD $\geq 6 \mathrm{~mm}, \mathrm{n}=1059)$, the baseline mean PPD was $6.5 \pm 0.5 \mathrm{~mm}$ which dropped to $2.6 \pm 0.7 \mathrm{~mm}$ by 12 months post-treatment and these initially deep pocket sites showed a mean PAL gain of $1.3 \pm 0.8 \mathrm{~mm}$.

\section{OHIP-14S}

The median OHIP-14S total score at baseline was 17 (maximum $=41$, minimum $=0$ interquartile range $=14$ ). This decreased slowly following the completion of non-surgical periodontal treatment, scoring a median of 16 (maximum $=42$, minimum $=1$, interquartile range $=15)$ immediately post-treatment, to 16 (maximum $=42$, minimum $=0$, interquartile range $=14)$ and 15 (maximum $=43$, minimum $=0$, interquartile range $=17$ ) at 1 -month and 3-month post-treatment respectively, then reached a median score of 14 (maximum $=45$, minimum $=0$, interquartile range $=15$ ) at 6 months; thereafter it remained at more or less same level not showing significant change, giving median score of 13 (maximum $=48$, minimum $=0$, interquartile range =16) at 12 months. The multiple time-point comparison revealed that the change in overall OHIP-14S scores over the study period was significant $(p=0.025$, Friedman 
test). Analyzed in their respective domains, significant changes over the study period were observed for "physical pain" (d2) ( $p=0.001)$, "psychological discomfort" (d3) $(p<0.001)$ and "psychological disability" (d5) $(p<0.001)($ Fig. 3) while no significant change over time was registered for "functional limitation" (d1) $(p=0.06)$, "physical disability" (d4) ( $p=0.451)$, d6: social disability (d6) $(p=0.398)$ and handicap (d7) ( $p$ $=0.753)$.

\section{Discussion}

The present case series reports the healing responses of 65 Chinese patients with moderate-to-advanced periodontitis following non-surgical periodontal therapy, as well as the associated changes in oral health-related quality of life (OHQoL) throughout a 12-month post-operation observation period. Since the current investigation it is not an intervention study with a control group, the current report does not provide definitive evidence for the effectiveness of non-surgical periodontal treatment in improving of OHQoL. After all, it is considered unethical to incorporate an untreated periodontitis group over 12 months to be control subjects for such a study.

Clinical outcomes of the present study were compatible with those of the 
classical studies using non-surgical periodontal therapy (Badersten et al. 1981, 1984 \& 1987). There was significant decrease in $\mathrm{P} 1 \%$ and $\mathrm{BOP} \%$ and a mean gain in PAL of $1.3 \pm 0.8 \mathrm{~mm}$ in sites with initial probing depth of $\geq 6 \mathrm{~mm}$. This is in line with the findings of earlier publications that the reported range of PAL improvement for sites $\geq 7 \mathrm{~mm}$ at baseline to be between 0.5 and $2.0 \mathrm{~mm}$ (Morrison et al. 1980, Badersten et al. 1984), indicating that in the present study the clinical response of the subjects to non-surgical periodontal therapy was favorable and comparable to prior reports.

The clinical improvements reported were observed through objective assessment of changes in the periodontal status following non-surgical periodontal treatment. This recording of changes was carried out in line with the conventional and predominant therapist-centered protocols of healing response interpretation after dental treatment (Buck \& Newton 2001). However, a trend has emerged which demands a change in direction towards patient-centered approaches to treatment and treatment outcomes. Merely improving clinical parameters like PPD reduction or PAL gain may not be sufficient if these improvements do not result in the establishment and maintenance of a functioning, disability free dentition for life. It is the improvement of the well-being and quality of life of the patients which should be the ultimate aim of healthcare professionals.

Among the few measures developed for assessing OHQoL (for review see Slade 
et al. 1998, Allen et al. 1999), GOHAI (Atchison \& Dolan 1990) and OHIP (Slade \& Spencer 1994) are among the most widely used measures and have had their properties confirmed (Locker 2004). These two measures have been compared by Locker and colleagues in 2001 and were found to be equally good. To make the OHIP questionnaire simple and easy to use, a short-form OHIP questionnaire was derived and validated, and was concluded to have comparable reliability and validity to the long-form OHIP (Slade 1997). For usage in Chinese populations, the OHIP-14S was translated from the original English version and validated in Chinese (Wong et al. 2002). OHIP-14S was therefore administered in the present study. It should be noted that the questionnaire intended to capture subjects' perception between recall appointments, i.e. over the past 3 months. Such time reference may be shorter than the usual administration of OHIP-14S, but is getting recognized to be helpful in other short term clinical studies as well, even when administered on daily basis (Ozcelik et al. 2007, Jowett et al. 2009). Nevertheless the reference time frame of OHRQoL gives an estimation of the general condition in the last six month. The overlapping reference interval allows a close monitoring of the spontaneous changes taking place through the course.

An earlier report by Slade (1998) concluded that OHIP captures both improvement and deterioration in health status, creating new complexities for 
conceptualizing and analyzing change in longitudinal studies. The sensitivity of OHIP to change has been assessed (Allen et al. 2001) and was found to be good when applied to edentulous subjects receiving different prosthetic treatment protocols. A later report from this research group indicated that variations in OHIP-14S in relation to six self-reported symptoms of periodontitis includes swollen, sore or receding gums, toothache, loose teeth and bad breath ( $\mathrm{Ng} \&$ Leung 2006a). Nevertheless, the corresponding sensitivity of the instrument to changes after periodontal therapy, however, is yet to be determined, hence the current results should be interpreted with caution. One other possible limitation of the use of OHIP-14S is that it is not a condition-specific measure, i.e. the reported improvement in OHQoL was not necessarily only due to the resolution of periodontitis in the patients followed-up. While OHIP demonstrated good discriminant validity in various domains correlated with the oral health condition, other parameters, e.g. number of filled (all caries filled or stabilized in subjects before study commencement) and number of standing teeth or replaced teeth, remained stable in the course of our study, indicating an otherwise stable oral condition except for improvement in periodontal health. Nevertheless, a condition specific OHQoL tool (condition specific-OIDP, Tsakos et al. 2010) validated in the Chinese language would be highly desirable and would allow the above limitations to be specifically addressed. 
Recently, negative effects of periodontal disease via perceived stigma were reported in a small cohort study (O'Dowd et al. 2010), indicating that there may remain potentially certain domains not covered by the Locker conceptual model on which the OHIP-14S is based. That proposition, however, requires further substantiation and hence the related patient-centered negative impact, even though potentially relevant, was not followed up in the current study.

The mean full-mouth PAL at screening of the present study group was $4.0 \pm 1.0$ $\mathrm{mm}$, range $2.7-6.4 \mathrm{~mm}$ and the baseline average OHIP-14S total score was 17.6. This appeared clearly higher than the corresponding average OHIP-14S total score of 4.4 for local population with healthy periodontal conditions or only slight periodontal attachment loss (defined as mean full-mouth PAL $\leq 2 \mathrm{~mm}$ ), while obviously lower than the average OHIP-14S of 24.2 in those with severe attachment loss (defined as mean full-mouth PAL $>3.0 \mathrm{~mm})(\mathrm{Ng} \&$ Leung 2006b). Hence the mean overall OHIP-14S score of the current cohorts fall in the range expected from a community-based study in the local population (Ng \& Leung 2006a).

Oral health-related quality of life was noted to have improved right after the 4 - 6 sessions of non-surgical periodontal therapy which had included oral hygiene instruction, scaling and root debridement under local anesthesia. This is reflected through the OHIP-14S score changes, which reduced gradually (indicating an 
improvement of oral health-related quality of life) from average of 17.6 at baseline until reaching its lowest level of 14.3 at 6 months post-treatment, an average score which remained at more or less same level until end of the study. Such a trend corresponded to the corresponding improvement in clinical parameters. Although the reduction of OHIP-14S score has a modest magnitude in comparison with the changes in the clinical parameters, there have been no reports to indicate the minimally important clinical difference for the present $\mathrm{OH}-\mathrm{QoL}$ tool, minimum required change in OHIP-14S applied to treated periodontitis subjects to indicate improved QoL, the difference was perceived and reported by the patients through the questionnaire responses, and hence the changes are probably much more relevant to the patients' daily life than changes in measurements of attachment levels.

The possible underlying reasons for the improved patient perception with respect to OHQoL after non-surgical periodontal treatment can be complex. The current study showed that out of the seven OHIP-14S subdomains, significant improvement in physical pain, psychological discomfort and psychological disability were observed while for the remaining four subdomains, no or marginally insignificant changes could be detected throughout the study period. Social disability and handicap subscales of OHIP-14S were reported earlier not to appear to be associated with PAL severity ( $\mathrm{Ng} \&$ Leung 2006a), and this might explain why these two subdomains 
remained unchanged following upon non-surgical therapy. However, regarding the remaining OHIP-14S subscales of functional limitation and physical disability that was previously reported to be associated with PAL severity, no significant or marginally insignificant improvements were observable over time, indicating that non-surgical mechanical periodontal therapy alone seemed not to be associated with improvements of such OHQoL subscales affected by periodontitis, or perhaps at least a larger sample size would be needed to prove whether functional limitation improvement could be associated with non-surgical periodontal therapy. Other dental treatment regimens are needed in order to attenuate the negative impact of periodontitis on at least the physical disability aspects of OHQoL.

The association between non-surgical therapy and change in magnitude of OHIP-14S subscales of physical pain, psychological discomfort and psychological disability improvements, is worthy of further attention. At the end of the study, subscales of physical pain, psychological discomfort and psychological disability were found to have plateaued at median/average scores of 3/2.9, 2/2.2 and 2/2.3 respectively, in comparison to median levels of 4, 3 and 3 at baseline (Fig. 3). Such levels appear higher than the corresponding average levels of 1.0, 0.3 and 0.5 in community-based subjects with minimal periodontal attachment loss (Ng \& Leung 2006a). Regarding the OHIP-14S subscale of physical disability which non-surgical 
periodontal therapy appeared unable to significantly address to, its average level remained highest at 2.7 or reduced to 2.5 towards the end of the study (data not shown). It is yet to be discovered what therapeutic approaches can have an effect and to what magnitude these OHIP-14S subscales could be improved. From the current circumstantial evidence, it would be unrealistic to expect complete reversal of all impaired OHQoL among periodontitis subjects to the level of normal unaffected individuals consequent to periodontal or other relevant dental treatment alone.

That continuous improvement in OHQoL was perceived among the studied patients implies the overriding importance of restoration of periodontal health in periodontitis patients. In addition to a possible improvement on physical comfort, with periodontal health restored, patients may gain confidence in maintenance of their previously periodontal compromised dentition, hence the improvement in psychological discomfort and psychological disability.

Longitudinal investigations on the impact of non-surgical periodontal treatment on patient-oriented outcomes are scarce, if any. To the best of our knowledge, the present study is the only one report with OHQoL followed over a complete course of non-surgical periodontal therapy and its healing and resolution period. On the other hand, there have been more short-term or cross-sectional studies. Needleman and colleagues (2004), D’Avila et al. (2005), Ozcelik et al. (2007), Bajwa et al. (2007), 
and more recently Saito et al. (2010) and Tsakos et al. (2010) all reported improvement of OHQoL shortly after delivery of non-surgical periodontal treatment. Although these studies had relatively short follow-up periods and may not be able to represent the full effect of resolution of periodontal inflammation on OHQoL, they are nevertheless echoed by the findings of our current study.

Median OHIP-14S score appeared to improve compared to baseline at 3-month post-treatment and continued to decline giving rise to an overall statistically significant difference of OHQoL. Along with gain in PAL, non-surgical periodontal treatment was shown to help improve the patients' OHQoL. At 12 months post-treatment, the mean OHIP-14S was 14.3 , which is still much higher than the mean score of 4.4 reported among subjects with relatively healthy periodontal conditions (Ng \& Leung 2006a). Such results echo the findings by Jowett and colleagues (Jowett et al. 2009), who studied 20 patients with chronic periodontitis and 16 healthy subjects and compared their OHIP-14S through daily telephone interviews. The latter study concluded that with root surface debridement, there was significantly favorable impact on OHQoL in periodontal patients which could be sustained at review, but that the post-treatment OHQoL level remained higher than that of healthy subjects. This, together with our present study, reinforces the conclusion drawn earlier by our group ( $\mathrm{Ng} \&$ Leung 2006a) that loss of periodontal attachment is related to a 
deterioration in OHQoL. Since a controlled clinical trial of untreated periodontitis would not be ethical, it is not possible in this context to show a relationship between progressive loss of periodontal attachment and deterioration in OHQoL.

A long observation period as in the present study could be used to indicate by how much periodontal treatment would reverse the impact of periodontal destruction on OHQoL given full treatment response to the non-surgical intervention. However, it should be pointed out that, even with well-established psychological social measurement instruments like OHIP, the analysis is based on a scoring system with empirically assigned levels. Therefore, we may not be able to directly translate how much a statistically significant mild drop in the OHIP-14S score, as in this study, is equivalent to each individual's subjective perception and value on OHQoL.

The significance of this study does not merely lie in the level of post-treatment OHIP-14S achieved. Instead, it demonstrates the positive impact of non-surgical periodontal treatment from patients' perspectives, in addition to the measurable improvements in clinical parameters. This implies that non-surgical periodontal treatment, in addition to being an effective protocol in managing periodontal disease, is also rewarding to patients both clinically and psychosocially over a 12-month period. In turn, the delivery of non-surgical periodontal therapy is rewarding to the operator since the treatment plays a role in improving patient's well-being, and does 
not solely target the goal of achieving "merely the absence of disease and infirmity".

This would also add to the justification for subjects receiving periodontal treatment who have to bear with procedures which inevitably bring about transient or temporary pain and discomfort, such as pain and discomfort during and after therapy, and dentine hypersensitivity post-treatment. Nevertheless, the study also provides an opportunity for clinicians to recognize that there are areas in oral health related life quality, e.g. functional limitation and physical disability, upon which the treatment provided by them is perhaps less able or unable to impact. Further investigations and treatment strategy developments are needed to address fully the negative impacts of periodontitis upon these OHQoL subscales.

\section{Conclusions}

The relationship between non-surgical periodontal treatment and oral health-related quality of life has been demonstrated through a longitudinal case series study undertaken over a period of 12 months. Among these 65 non-smoking Chinese adults with moderate-to-advanced chronic periodontitis, healing response following non-surgical periodontal therapy was satisfactory in terms of decreases in $\mathrm{P} 1 \%, \mathrm{BOP} \%$, PPD and gain in PAL, indicating good efficacy of the non-surgical mechanical 
periodontal therapy delivered. At the same time, significant improvements of OHQoL following non-surgical periodontal therapy among the subjects in particular subscales regarding physical pain, psychological discomfort and psychological disability, were shown by the multi-time point administrations of the reliable and validated psychosocial measure, OHIP-14S, over the 12-month follow-up period. The median OHIP-14S score or specifically three subscales, physical pain, psychological discomfort and psychological disability, declined steadily in the first 6 months post-treatment, and remained at lower levels over the subsequent visits. It may therefore be concluded that successful non-surgical periodontal therapy is associated with positive impact on OHQoL in regard to physical pain, psychological discomfort and psychological disability in patients with moderate-to-advanced chronic periodontitis. This shows that while loss of periodontal attachment may not be reversed, control of periodontal disease and its stability could possibly be appreciated by patients at a personal level, which may be very relevant given the contemporary concept of holistic healthcare taking precedence over operator-dominated assessments of healing reflected only by improvement in clinical parameters. This report together with our previous study ( $\mathrm{Ng} \&$ Leung 2006a) shows that periodontitis and its non-surgical treatment probably conferred no impact on perceived social disability and handicap, however, other treatment strategies would be required to help improve 
perceived OHQoL especially in terms of physical disability. Further studies are warranted to identify the best patient-centered treatment options that can minimize the negative effects of periodontitis on perceived social disability and handicap and perceived physical disability which remain unchanged despite successful non-surgical periodontal therapy.

\section{Acknowledgment}

The work described in this paper was partially supported by grants from the Research Grants Council of the Hong Kong Special Administrative Region, China (HKU 772110M) and The University of Hong Kong Research Committee grant: 10208714.

\section{References}

Adulyanon, S. \& Sheiham, A. (1997) Oral impacts on daily performances. In: Measuring Oral Health and Quality of Life. Slade, G.D. editor. Chapel Hill: University of North Carolina, Dental Ecology, pp. 151-160.

Allen, P. F. (2003) Assessment of oral health related quality of life. Health and Qualaity of Life Outcomes 1, 40. 
Allen, P. F., McMillan, A. S. \& Locker, D. (2001) An assessment of sensitivity to change of the Oral Health Impact Profile in a clinical trial. Community Dentistry and Oral Epidemiology 29, 175-182.

Allen, P. F., McMillan, A. S., Walshaw, D. \& Locker, D. (1999) A comparison of the validity of generic- and disease-specific measures in the assessment of oral health-related quality of life. Community Dentistry and Oral Epidemiology 27, 344-352.

Atchison, K. A. \& Dolan, T. A. (1990) Development of the Geriatric Oral Health Assessment Index. Journal of Dental Education 54, 680-687.

Åslund, M., Suvan, J., Moles, D. R., D’Aiuto, F., \& Tonetti, M. S. (2008) Effects of two different methods of non-surgical periodontal therapy on patient perception of pain and quality of life: A randomized controlled clinical trial. Journal of Periodontology 79, 1031-1040.

Badersten, A., Nilveus, R. \& Egelberg, J. (1981) Effect of nonsurgical periodontal therapy. I. Moderately advanced periodontitis. Journal of Clinical Periodontology 8, 57-72.

Badersten, A., Nilveus, R. \& Egelberg, J. (1984) Effect of nonsurgical periodontal therapy. II. Severely advanced periodontitis. Journal of Clinical Periodontology 11, 63-76. 
Badersten, A., Nilveus, R. \& Egelberg, J. (1987) Effect of nonsurgical periodontal therapy (VIII). Probing attachment changes related to clinical characteristics. Journal of Clinical Periodontology 14, 425-432.

Bajwa, A., Watts, T. L. \& Newton, J. T. (2007) Health control beliefs and quality of life considerations before and during periodontal treatment. Oral Health and Preventive Dentistry 5, 101-104.

Bernabé, E. \& Marcenes, W. (2010) Periodontal disease and quality of life in British adults. Journal of Clinical Periodontology 37, 968-972.

Buck, D. \& Newton, J. T. (2001) Non-clinical outcome measures in dentistry: publishing trends 1988-98. Community Dentistry and Oral Epidemiology 29, 2-8.

D'Avila, G. B., Carvalho, L. H., Feres-Filho, E. J., Feres, M. \& Leao, A. (2005) Oral health impacts on daily living related to four different treatment protocols for chronic periodontitis. Journal of Periodontology 76, 1751-1757.

Dental Service Head Office. (2002) Oral health survey 2001: common dental diseases and oral health related behavior. Hong Kong SAR: Department of Health.

Farquhar, M. (1995) Definitions of quality of life: a taxonomy. Journal of Advanced Nursing 22, 502-508.

Giddon, D. B. (1987) Oral health and the quality of life. Journal of the American 
College of Dentists 54, 10-15.

Jowett, A. K., Orr, M. T., Rawlinson, A. \& Robinson, P. G. (2009) Psychosocial impact of periodontal disease and its treatment with 24-h root surface debridement. Journal of Clinical Periodontology 36, 413-418.

Locker, D. (2004) Oral health and quality of life. Oral Health and Preventive Dentistry 2 Suppl 1, 247-253.

Locker, D., Matear, D., Stephens, M., Lawrence, H. \& Payne, B. (2001) Comparison of the GOHAI and OHIP-14 as measures of the oral health-related quality of life of the elderly. Community Dentistry and Oral Epidemiology 29, 373-381.

Maslow, A. H. (1970) Motivation and Personality New York: Harper \& Row.

McGrath, C. \& Bedi, R. (2001) Can dental attendance improve quality of life? British Dental Journal 190, 262-265.

McGrath, C. \& Bedi, R. (2002) Population based norming of the UK oral health related quality of life measure (OHQoL-UK@). British Dental Journal 193, $521-524$.

McGrath, C. \& Newsome, P. R. (2007) Patient-centred measures in dental practice: 2. Quality of life. Dental Update 34, 41-42, 44.

Morrison, E. C., Ramfjord, S. P. \& Hill, R. W. (1980) Short-term effects of initial, 
nonsurgical periodontal treatment (hygienic phase). Journal of Clinical Periodontology 7, 199-211.

Needleman, I., McGrath, C., Floyd, P. \& Biddle, A. (2004) Impact of oral health on the life quality of periodontal patients. Journal of Clinical Periodontology 31, 454-457.

Ng, S. K. \& Leung, W. K. (2006a) Oral health-related quality of life and periodontal status. Community Dentistry and Oral Epidemiology 34, 114-122.

Ng, S. K. \& Leung, W. K. (2006b) A community study on the relationship between stress, coping, affective dispositions and periodontal attachment loss. Community Dentistry and Oral Epidemiology 34, 252-266.

Ng, S. K. \& Leung, W. K. (2008) A community study on the relationship of dental anxiety with oral health status and oral health-related quality of life. Community Dentistry and Oral Epidemiology 36, 347-356.

O’Dowd, L. K., Durham, J., McCracken, G. I. \& Preshaw, P. M. (2010) Patients' experiences of the impact of periodontal disease. Journal of Clinical Periodontology 37, 334-339.

Oliver, R. C., Brown, L. J. \& Loe, H. (1998) Periodontal diseases in the United States population. Journal of Periodontology 69, 269-278.

Ozcelik, O., Haytac, M. C. \& Seydaoglu, G. (2007) Immediate post-operative effects 
of different periodontal treatment modalities on oral health-related quality of life: a randomized clinical trial. Journal of Clinical Periodontology 34, 788-796.

Saito, A., Hosaka, Y., Kikuchi, M., Akamatsu, M., Fukaya, C., Matsumoto, S., Ueshima, F., Hayakawa, H., Fujinami, K. \& Nakagawa, T. (2010) Effect of initial periodontal therapy on oral health-related quality of life in patients with periodontitis in Japan. Journal of Periodontology 81, 1001-1009.

Slade, G. D. (1997) Derivation and validation of a short-form oral health impact profile. Community Dentistry and Oral Epidemiology 25, 284-290.

Slade, G. D. (1998) Assessing change in quality of life using the Oral Health Impact Profile. Community Dentistry and Oral Epidemiology 26, 52-61.

Slade, G. D. \& Spencer, A. J. (1994) Development and evaluation of the Oral Health Impact Profile. Community Dental Health 11, 3-11.

Slade, G. D., Strauss, R. P., Atchison, K. A., Kressin, N. R., Locker, D. \& Reisine, S. T. (1998) Conference summary: assessing oral health outcomes--measuring health status and quality of life. Community Dental Health 15, 3-7.

Tsakos, G., Bernabé, E., D’Aiuto, F., Pikhart, H., Tonetti, M. S., Sheiham, A. \& Donos, N. (2010) Assessing the minimally important difference in the Oral 
Impact on Daily Performances index in patients treated for periodontitis. Journal of Clinical Periodontology 37, 903-909.

Wan, C. P., Leung, W. K., Wong, M. C. M., Wong, R. M. S., Wan, P., Lo, E. C. M. \& Corbet, E. F. (2009) Effects of smoking on healing response to non-surgical periodontal therapy: a multilevel modelling analysis. Journal of Clinical Periodontology 36, 229-239.

Wong, M. C., Lo, E. C. \& McMillan, A. S. (2002) Validation of a Chinese version of the Oral Health Impact Profile (OHIP). Community Dentistry and Oral Epidemiology 30, 423-430.

World Health Organization (1948) Preamble to the Constitution of the World Health Organization as adopted by the International Health Conference, New York, 19-22 June, 1946. Official Records of the World Health Organization, no. 2, p. 100. www.who.int/about/definition/en/print.html 


\section{Legend}

Figure 1. Study design

Figure 2. Mean (SD) periodontal parameters of subjects from baseline to 12 months post-treatment. (A) $\mathrm{Pl} \%$ and $\mathrm{BOP} \%$ profile over the study period, (B) PPD profile, in shallow, moderate and deep pockets categories, over the study period, (C) Change of PAL over the study period. ( $p<0.0001$ vs baseline, repeated measures ANOVA).

Figure 3. Median OHIP-14S (A) and selected subscales scores (B) of subjects from baseline to 12 months post-treatment. OHIP-14S, subscales d2: physical pain, d3: psychological discomfort, and d5: psychological disability significantly improved over time ( $p \leq 0.025$, Friedman test). Subscales d4: physical disability, d6: social disability and d7: handicap changes (not shown) over study period were not significant ( $p \geq 0.398$ ); subscale d1: functional limitation changes (not shown) over study period was marginally insignificant ( $p=0.06$, Friedman test). 
Table 1. Subject-level clinical parameters over study period

\begin{tabular}{cccccc}
\hline & & \multicolumn{4}{c}{ Months post-treatment } \\
\cline { 3 - 6 } & Baseline & 3 & 6 & 9 & 12 \\
\hline Full-mouth mean PPD (mm) & $3.25 \pm 0.70$ & $2.06 \pm 0.32$ & $1.91 \pm 0.31$ & $1.84 \pm 0.25$ & $1.75 \pm 0.23$ \\
Percentage proportion sites with baseline PPD of & & & & \\
$0-3 \mathrm{~mm}$ & 62.9 & 91.4 & 93.6 & 94.8 & 95.6 \\
$4-5 \mathrm{~mm}$ & 25.9 & 7.2 & 5.2 & 4.4 & 3.5 \\
$\geq 6 \mathrm{~mm}$ & 11.2 & 1.4 & 1.2 & 0.8 & 0.8 \\
\hline
\end{tabular}


6

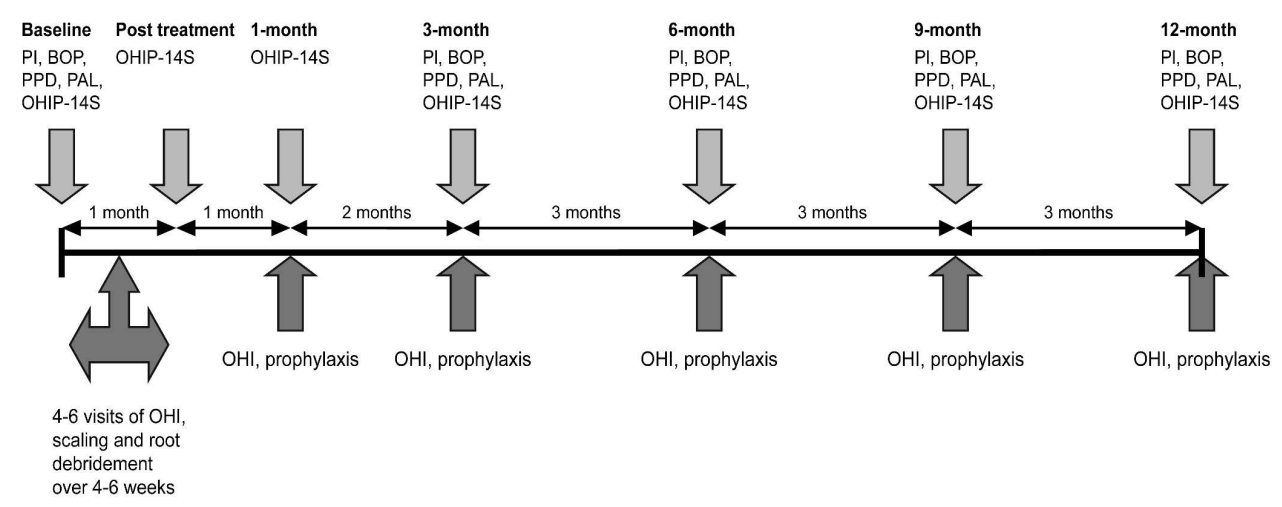

$182 \times 68 \mathrm{~mm}(600 \times 600 \mathrm{DPI})$ 

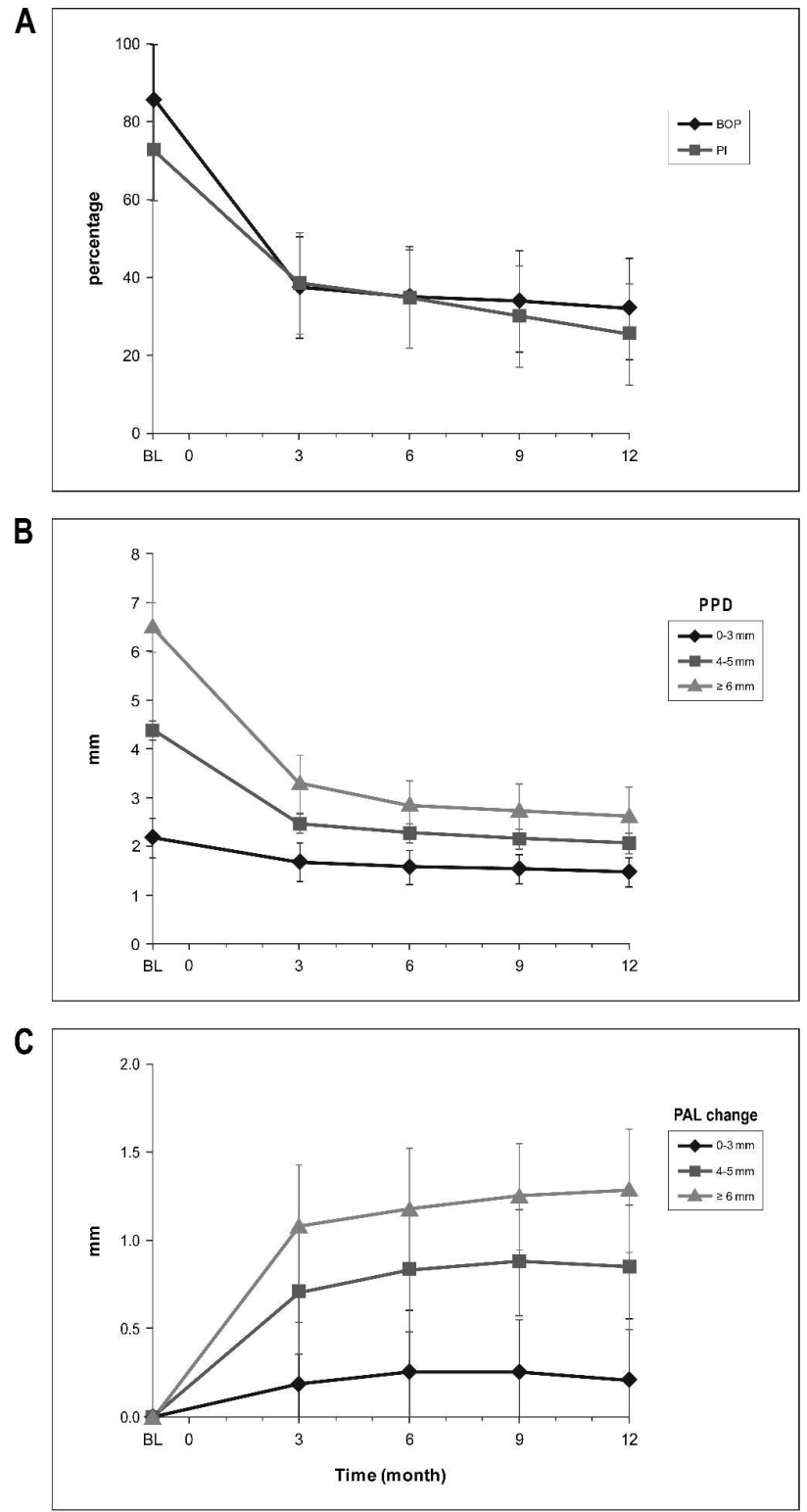

$133 \times 253 \mathrm{~mm}(600 \times 600 \mathrm{DPI})$ 


\section{OHIP-14S}

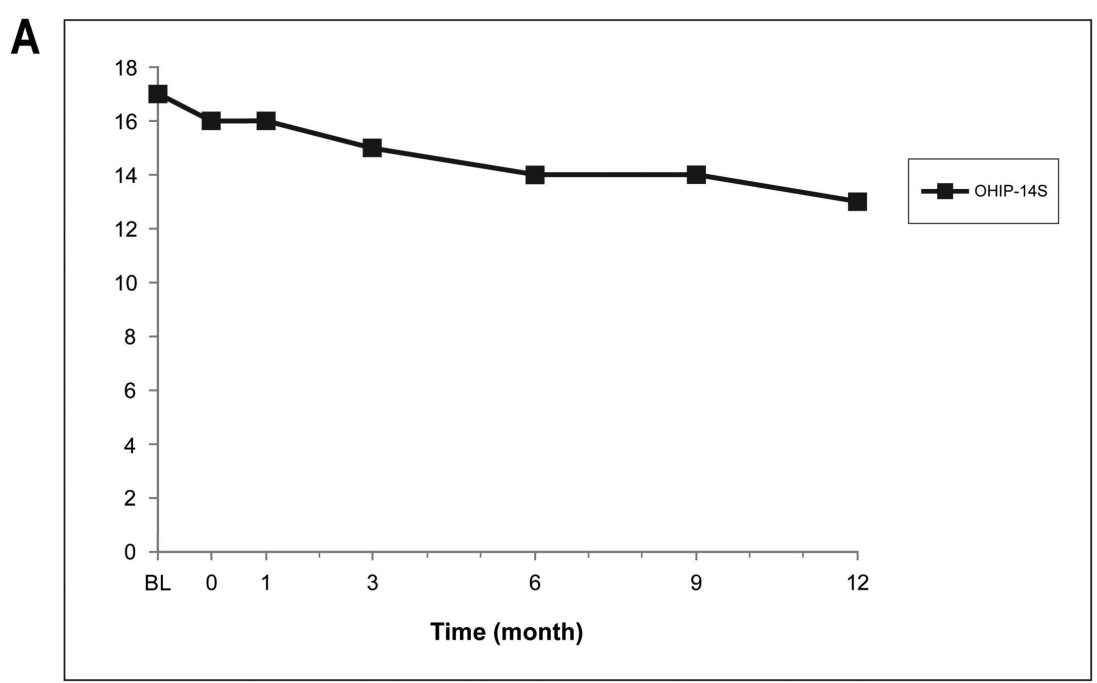

OHIP-14S domains

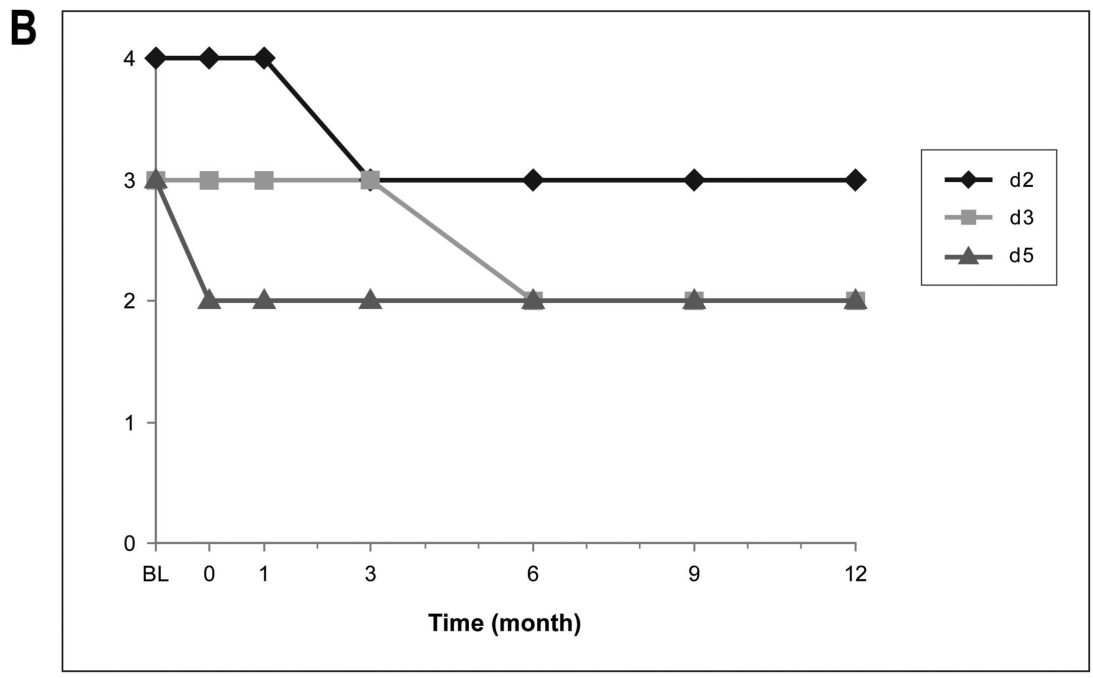

$188 \times 266 \mathrm{~mm}(300 \times 300 \mathrm{DPI})$ 